\title{
FIRST EXPERIENCE WITH THE PETRA CONTROL SYSTEM
}

Hans Frese, Uwe Gloer, Herbert Haller, Gerd Hochweller

Kurt Holm, Jörg Kewisch, Wolfgang Krechlok, Jürgen Maass

Franz Peters, Rüdiger Schmitz, Reinhard Stadtmiller,

Holger Wagner

Deutsches Elektronen-Synchrotron DESY

Notkestr. 85, 2000 Hamburg 52, W. Germany

\section{Summary}

This paper describes the overal1 setup and struture of the PETRA Control System. Experience with the system during construction and first running periods are reported. Typical applications in the fields of vacuum, magnets, monitors, and RF illustrate the operational possibilities which the control system provides for 'normal' users, who are not computer professionals. Possibilities for future developments are mentioned.

\section{Introductions}

PETRA (1) is an $e^{+}-e^{-}$-storage ring with a maximum energy of $19 \mathrm{GeV}$. Injection energy is $7 \mathrm{GeV}$, filling time is 15-20 minutes, energy ramping takes $\sim 200 \mathrm{sec}-$ onds, and the lifetime of the colliding beams is several hours.

The control system has to handle the

magnets: 270 power supplies for main magnets, correction coils, injection, etc.

monitors: 200 supply and control units for beam position, beam profile and other monitors

vacuum: 800 vacuum power supplies and control de$v$ ices for sputter pumps, mass spectrometer, valves, pressure gauges etc.

RF et al.: 64 cavities, eight clystrons (500 MHz) with their associated equipment, status control of personnel and machine interlock systems.

\section{Structure of the Control System}

Fig. 1 shows the hardware structure. Three identical consoles are available to the operators in the central control room, one dedicated to monitors and magnets, one for RF components, and one for vacuum. Besides a standardized set of man machine communication devices (2), each console may contain an oscilloscope for real time signals, TV monitors for display of camera pictures, plus interlock and intercom equipment. (Fig. 3)

The storage ring component supplies and control units are concentrated in substations around the ring in the 6 experimental, $4 \mathrm{RF}$ and one main magnet power supply ha11.

The equipment is connected to the computers by a specially designed Serial Data Acquisition Control system (SEDAC) (3).

\section{Software Structure}

Exclusive computer control was one of PETRA's design objectives. Consequently, numerous control devices have no manual control features at all. This made operational computer hardware and software mandatory at the time of delivery of the first piece of accelerator equipment. Because of the lack of computer professionals, most of the test programs had to be written by the equipment designers themselves. Realization was made possible, since we both had a working operating system (SINTRAN III delivered by the computer manufacturer(4)) and an easy-to-learn high level interactive computer language especially suited for this type of application. (NODAL, a CERN SPS development (5))

New monitor calls were added to the system to handle PETRA hardware - both the man machine communications and the process I/O via SEDAC - in away consistent with the SINTRAN III standards (6). Communication with the hardware components is established by means of Equipment Functions (EQF). Each type of hardware component is handled by a unique EOF, which takes care of hardware bit layouts and translation of devices specific 'count ticks' into standard units of measurement, etc. The basic part of NODAL (interpreter, arithmetic package, and others) together with the application specific set of equipment functions plus the routines to handle the man machine communications constitute the PETRA Operator Command Language (POCAL).

Al1 operating programs are written in POCAL. Fig.2 portraits the software/hardware structure.
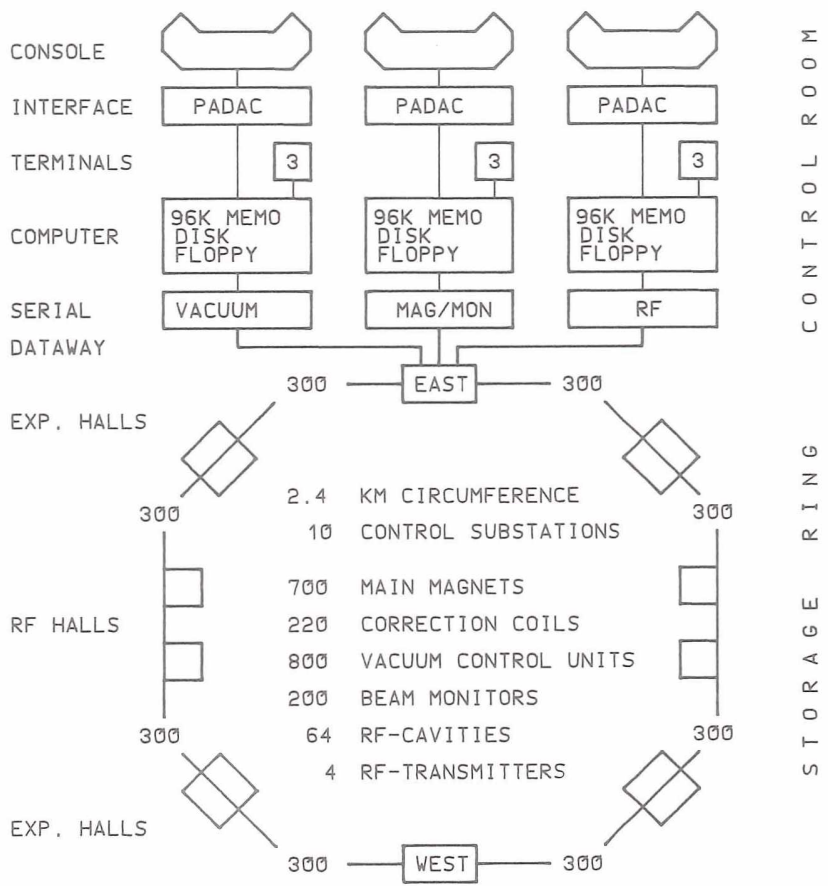

FIG, 1 STRUCTURE OF THE PETRA CONTROL SYSTEM

\section{Set up Experience}

Including the control system, PETRA had to be built by DESY's existing personnel. In the field of controls, only mass production and cabling were done by outside firms.

In summer 1975, a collaboration of members from several DESY groups was formed to develop and realize

- the central control room

- the control system software

- the substations, including cabling

- the safety and intercom systems

- most of the beam monitors

Maximum membership was $~ 40$ people; since $80 \%$ of these 
could work for PETRA only $50 \%$ of their time, this amounts to a control group of 24 .

In sumer 76 the first computer was delivered and software and hardware development started. The next steps were:

- spring 77: first positron beam injected into a part of PETRA

- spring 78: half of the machine installed and tested with beam (electrons and positrons)

- summer 78: both beams stored.

For economic reasons, our manufacturers performed only visual inspection of mass produced SEDAC modules; 1aboratory tests were done at DESY after delivery:

$30 \%$ needed minor, $10 \%$ major repairs. Long term testing was performed after installing the modules. This was made possible by two features:

- SEDAC electrical, physical and logical specifi-

cations, which allow the addition and replacement of modules without disturbing the already working part of the control system and the SEDAC system itself.

- a multi-user operating system which provided execution of program development, hardware test and operating in parallel without interference.

There was early feedback between operation and development by simultaneous set up of hardware and its associated software. The equipment designers were able to test their own hardware modules using terminals in the substations. The test programs communicated with the hardware via the same equipment functions ( $E Q F^{\prime} \mathrm{s}$ ) to be used in the operating programs, providing an early test of the software/hardware interface.

Thus, testing a new substation and incorporating it into the control system was accomplished within a few days. Running operating programs in a time-sliced environment provided adequate response times. Before handing over the control system to the operation crew, we switched to a real time version of POCAL with allows the execution of several POCAL programs at different priorities and provides all of these programs access to common data bases.

\section{Operating Examples}

Vacuum: Currently, the control system handles 800 devices such as sputter pumps, valves, massspectrometers, pressure gauges etc. Normal1y, an overview ot the vacuum pressures is displayed which is updated at regular intervals. Data collection, calculation and preparation of this display (see Fig.5) takes 10 seconds for 450 individual pumps. In addition to the pressure, pump and valve status, error message etc. are displayed. Using tracker ball and cursor the operator may displayed on the second screen three neighbouring vacuum sections in more detail (Fig.6). This part of the program provides manipulation possibilities for individual functions of the displayed components. Together with features such as collective operations, limit chechs, etc. The vacuum control system is considered complete and has been handed over to the vacuum group (7).

Magnet and Monitors: 270 magnet power supplies with different accuracies and 200 beam control monitors are managed by the control system. Fig. 6 portrays one of the displays generated by the program used most frequently in this field.

The upper part of the screen is dedicated to magnets, alternatively displaying

- a11 main magnets

- correction coils (one quadrant at a time)

- injection region.

Individual and collective operations are provided, including

- on line calculation of compensated bump operation with three coils any in a quadrant. Actual optical and physical parameters of the coils are displayed (Fig. 7 ).

- energy ramping (ball varied or target)

- transitions between different sets of machine optics with stored beam.

Monitors are displayed on the lower half of the same screen divided into

- beam position and fluorescent screen monitors (one quadrant at a time)

- beam profile monitors

- collimators and scrapers Operating includes

- local and global beam position measurements

- TV signal switching for the real time displays

- screen positioning.

More detailed versions of either the magnet or monitor displays can be generated on the second screen of this console. All associated operation interventions are triggered using tracker ball, cursor, and menu fields on the screens (8).

$\mathrm{RF}$ : The RF programs use the split screen technique to display the status of the 8 klystrons with their associated equipment and of 32 (64) cavities. Supported operating possibilities include the cold start sequence for the transmitters (9). (Fig. 4

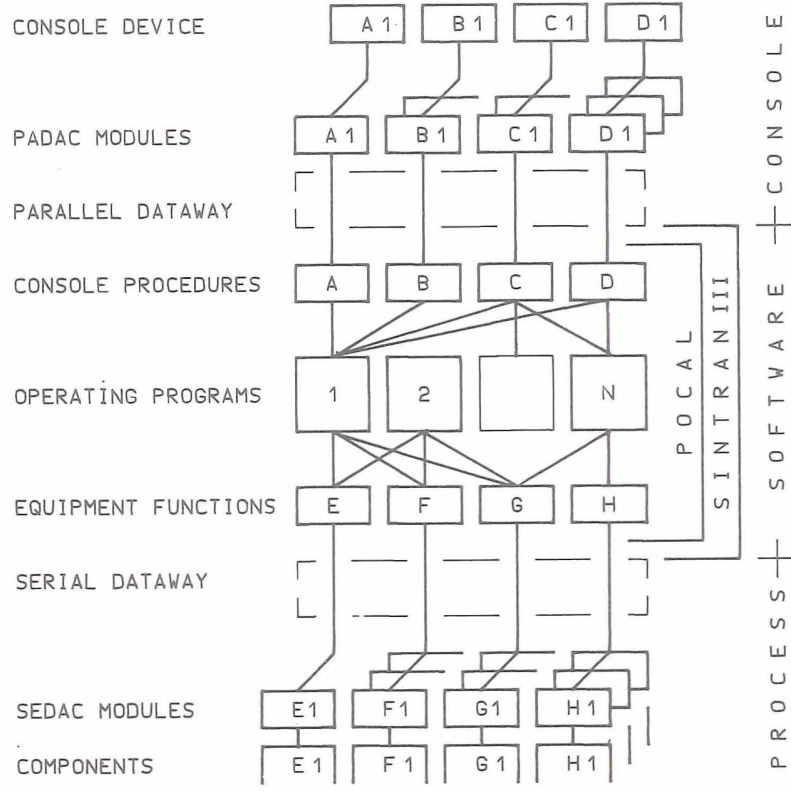

FIG 2. SOFTWARE / HARDWARE STRUCTURE

\section{Conclusions}

The operating of each of PETRA's components completely depends on the existence and usability of the associated control system parts. Although PETRA's construction run ahead of schedule by several months the control system development and installation kept pace. At no time we were held up by bottlenecks in the control system.

Using an interpreter proved to be very helpful, especially during the set up phase, when speed of realization was more important then speed of execution. For actual operations faster response was necessary. It was achieved by implementing more complex POCAL functions, which reduce the interpreter overhead significantly. Further improvements are envisaged by using a POCAL compiler. 
Experience has shown that PETRA can be operated with the control system configuration shown in Fig. 1. How ever, since computers and consoles are available for maintenance and program development (using the console devices) only during the short PETRA shut down periods, we are currently installing a fourth identical set up. Future plans include the development of a computercommunication system and the extension of the control system to incorporate the other existing accelerators on the DESY site.

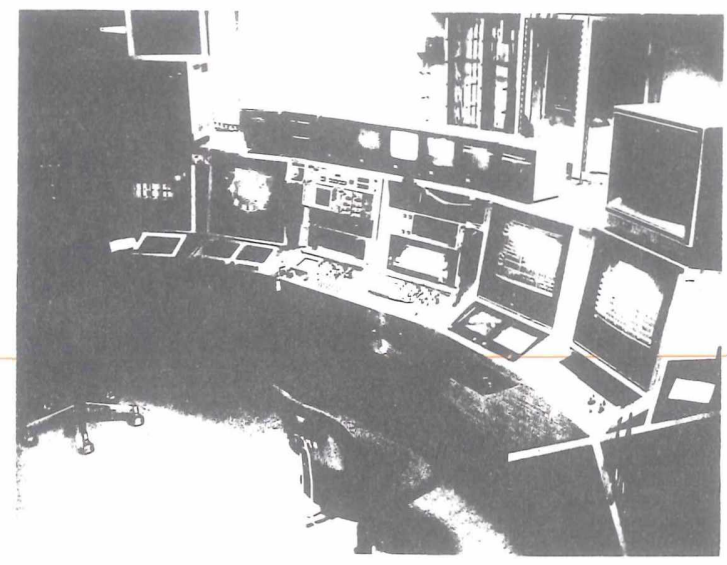

Fig.3 Two of three consoles

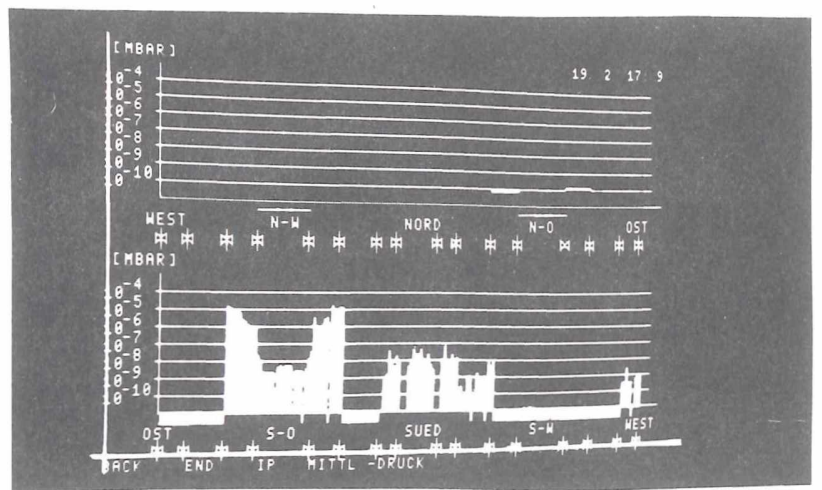

Fig. 5 Vacuum overview

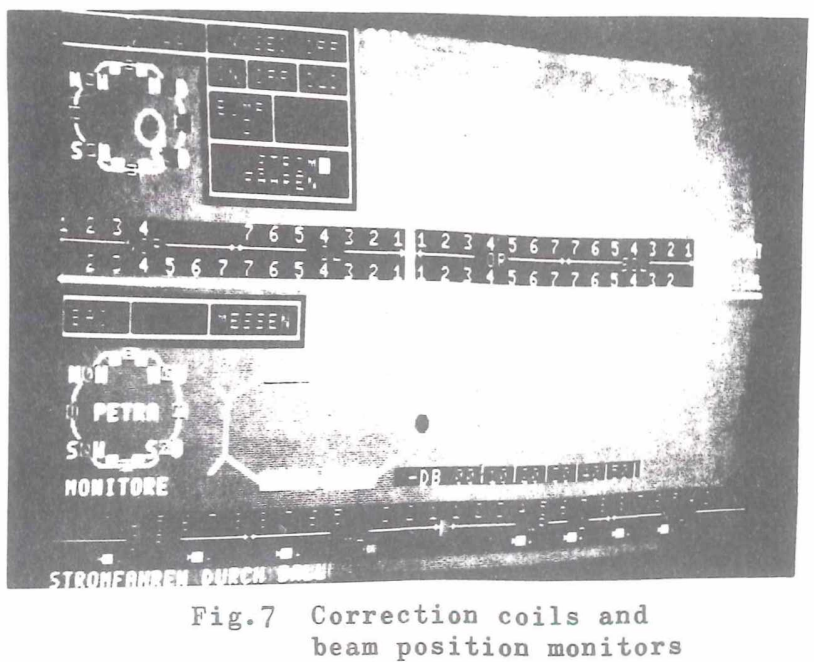

\section{References}

1) G.A. Voss in this proceedings

2) G. Hochweller in this proceedings

3) H. Frese in this proceedings

4) Norsk Data, Box 4 Lindeberg gard, Oslo 10

5) M.c. Crowley-Milling and G. Shering, The NODAL system for the SPS. CERN 78-07

6) W. Krechlock, R. Schmitz, DESY-Report to be published

7) H. Wagner et al, DESY-Report to be published

8) J. Kewische et al, DESY-Report to be published

9) H. Haller et al, DESY-Report to be published

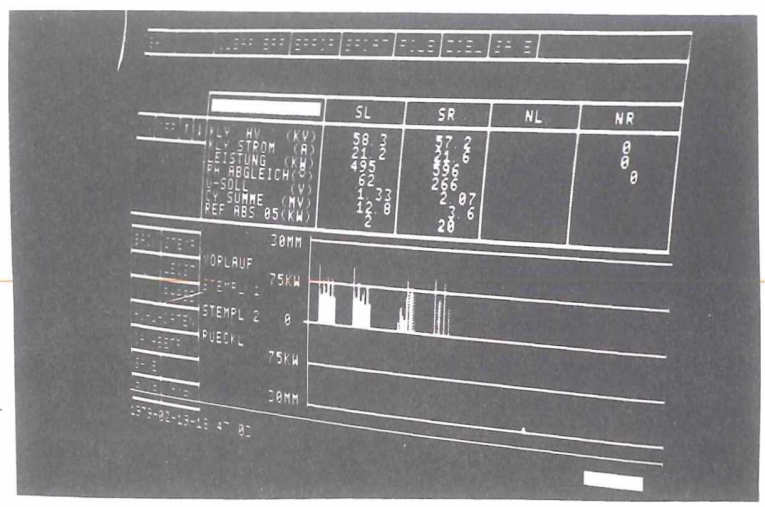

Fig.4 RF operating display

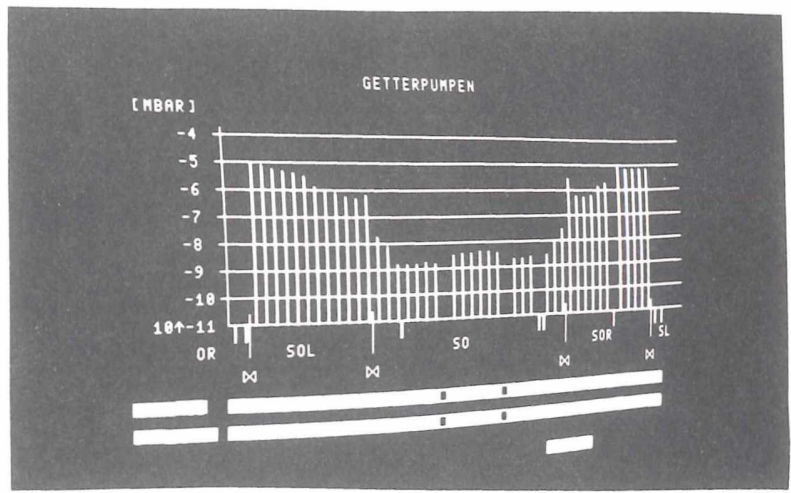

Fig.6 More details(three sections)

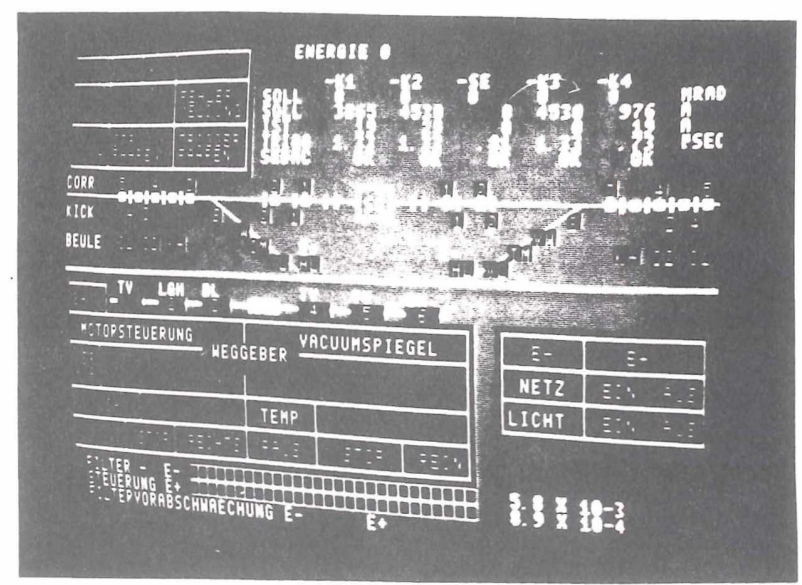

Fig. 8 Injection region and profil monitors 